\title{
NGC 5128: The Giant Beneath
}

\author{
Gretchen L. H. Harris \\ Department of Physics and Astronomy, University of Waterloo, Waterloo ON, N2L 3G1, Canada. \\ Email: glharris@astro.uwaterloo.ca
}

Received 29 September 2009, accepted 12 April 2010

\begin{abstract}
This paper reviews what has been learned about the old stellar population of NGC 5128, the only large elliptical galaxy close enough that we can currently observe individual stars as faint as the horizontal branch. Although its galaxy type is still a matter of debate, the uncertainties over distance are now largely resolved; comparison of five stellar distance indicators gives $d=3.8 \pm 0.1 \mathrm{Mpc}$. The globular cluster system, which was once perplexingly invisible, is now known to be predominantly old with a substantial metal-rich component. The globular cluster system (GCS) total population and luminosity function are normal and the clusters follow the same fundamental plane relation as those in the Milky Way and M31. Finally, the halo out to at least $\sim 7 r_{\text {eff }}$ is dominated by metal-rich stars which are also predominantly old, with age and metallicity tantalizingly similar to the majority of globular clusters.
\end{abstract}

Keywords: Galaxies: distances — galaxies: stellar content and star clusters — galaxies: individual (NGC 5128)

\section{Introduction}

In this review, early problems that confused our understanding of NGC 5128 are discussed and an overview of what we have learned about the properties of its old stars is provided, with an emphasis on the globular cluster (GC) and field star populations. Because NGC 5128 is $<4 \mathrm{Mpc}$ away $\left((m=M)_{0}=27.9\right)$, we can study its stellar component in greater depth and detail than is possible for any other large elliptical (E) galaxy. Consequently, we have data for hundreds of GCs and planetary nebulae that tell a rich history of how and when its stars formed. In addition (and unknown to many readers) we can now resolve individual halo stars, long-period variables (LPV), and Cepheids in NGC 5128, telling us its distance and providing additional clues to its history. For recent results on these and other stellar components see: distance (Harris et al. 2010); ages of field stars and LPV (Rejkuba et al. 2003, 2005); GCs (Peng, Ford \& Freeman 2004b; Woodley \& Gomez 2010b; Woodley et al. 2010a); and planetary nebulae (Walsh 1999; Peng, Ford \& Freeman 2004a; Walsh et al. 2010).

\section{The Nearby E Galaxy}

When most people look at NGC 5128 they see a peculiar galaxy dominated by a prominent dust lane and ignore the rest. What I see is a nearby E galaxy whose oldest stars can tell us a great deal about its history. A quick look at the literature over the past 60 years shows a growing body of research, mostly on the radio jets, dust lane, and X-ray sources, and much less on the dominant baryonic component which is the E galaxy beneath. But, particularly in the past 20 years, we are now beginning to understand the stellar history of NGC 5128 as well.

\subsection{Galaxy Type}

NGC 5128 continues to be perceived as a galaxy whose fundamental properties are likely to be atypical because it is associated with a strong radio source and its physical appearance in the centre is dominated by a wide absorption band. But studies as early as the mid-1950s indicated that, aside from the dust lane, the global structural properties of NGC 5128 were consistent with those of an E galaxy. Baade \& Minkowski (1954) called it 'an unresolved E0 nebula with an unusually strong and wide central absorption band' and concluded that 'the two nebulae ... form a close pair in a state of strong gravitational interaction, perhaps actually in collision'. Based on data from photographic plates in three wavelength bands, Sérsic (1958) found isophotal countours which were remarkably similar in all colours and fitted a deVaucouleurs profile out to $10 \mathrm{arcmin}(\sim 10 \mathrm{kpc})$ from the centre of the galaxy.

In their review, Ebneter \& Balick (1983) argue that 'Cen A has a probably undeserved reputation for being one of the most peculiar galaxies in the sky' and point out that many of its characteristics would not be as visible or obvious if the system were more distant. Graham (1979), in his study of both the halo light distribution and the dynamics of the dust lane region, concludes that 'the main body of NGC 5128 resembles in many respects a normal giant elliptical galaxy' and 'the main radio characteristics of NGC 5128 and the energetic phenomena ... 
are a consequence rather than the cause of the unusual structural features of the galaxy as a whole'.

There is still no clear consensus on the galaxy type classification for NGC 5128. As seen in Table 1, the 'vote' is clearly split between S0p and E0p, presumably because of the dust lane. Morgan (1958) suggested that the S0 classification is applied to galaxies which are often very different from each other and van den Bergh (1990) agrees that 'the S0 classification type comprises a number of physically quite distinct types of objects that exhibit only superficial morphological similarities'. More recently, van den Bergh (2009 private communication) indicates that 'NGC 5128 is an object that does not find a natural home in the Hubble classification scheme'. In this review paper, NGC 5128 will be considered an Ep galaxy.

\subsection{Distance}

Several decades passed before a reliable distance to NGC 5128 - a galaxy not far beyond the boundaries of the Local Group - could be determined, mainly because (until recently) the galaxy was too distant to apply most stellar candles, and too close to use the Hubble Law with confidence or to observe like other large E galaxies. In the past two decades, however, we have been able to derive a distance based on the properties of its stellar component.

As can be seen in Figure 1, distance estimates for NGC 5128 before $\sim 1980$ ranged from 2 to $9 \mathrm{Mpc}$ - a factor of $>4$. This apparent dichotomy between large and small distances often led to the use of an 'average' value of $\sim 5 \mathrm{Mpc}$. But since the late 1980s it has been possible to use a variety of stellar distance indicators: luminosity of the red giant branch tip (TRGB), planetary luminosity function (PNLF), long-period variables (LPV), surface brightness fluctuations (SBF), and Cepheid variables. The quality of the data and the calibration of these methods now support a distance of $3.8 \pm 0.1 \mathrm{Mpc}$ for NGC 5128 (see Harris et al. 2010, in this volume for a full review).

\section{The Globular Clusters}

The apparent lack of GCs in NGC 5128 was an important factor in the confusion about its nature. By the late 1970s, studies of galaxies beyond the Local Group had found GCs in every galaxy searched, and shown that large E galaxies contained thousands of GCs (see Harris \& Racine (1979)

Table 1. Galaxy type for NGC 5128

\begin{tabular}{ll}
\hline Galaxy type & Source \\
\hline Like NGC 3379 (E1) & Sérsic $(1958)$ \\
E0p & Morgan (1958) \\
$($ E0 + Sb) & Sandage (1961) \\
S0p & Freeman (1970) \\
E0p & van den Bergh (1976) \\
E + spiral & Dufour et al. (1979) \\
S0 & Sandage \& Tammann (1981) \\
S0p & NED database (2009) \\
Ep & This paper \\
\hline
\end{tabular}

and references therein). In the case of NGC 5128, however, no such GC population had been uncovered.

Van den Bergh (1979) carried out star counts in annuli between $45 \operatorname{arcsec}(\sim 800 \mathrm{pc})$ and $484 \operatorname{arcsec}(\sim 9000 \mathrm{pc})$ (see Figure 2), finding an excess of only $15 \pm 60$ objects above background. An obvious explanation for the apparent lack of GCs in NGC 5128 was the dust lane, and that this indicated a cataclysmic history. We now know that the GCs are present in numbers expected for an E galaxy in a small group (e.g., Harris et al. 1984; Harris, Harris \& Geisler

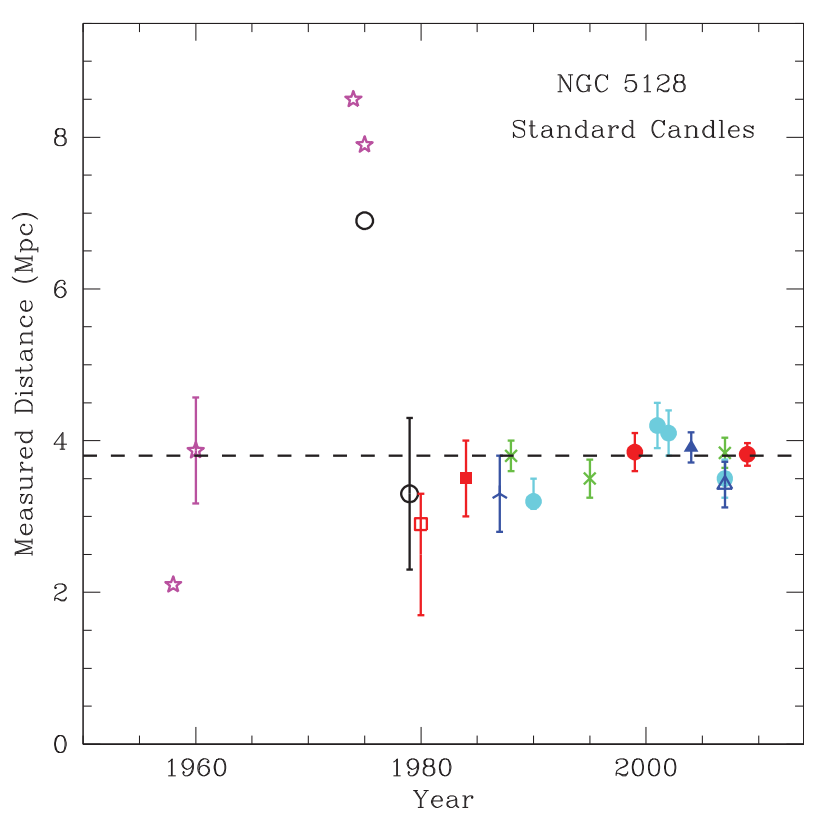

Figure 1 Distance to NGC 5128 vs. year of publication: HII regions (magenta stars), redshift (open black circles), GCs (red squares), SN1986g (blue chevron), TRGB (red circles), SBF (cyan circles), PNLF (green stars), LPV (filled blue triangle), and Cepheid variables (empty blue triangle). Error bars are plotted when available. For sources see Israel (1998), Rejkuba (2004), Harris et al. (2010) and references therein. The horizontal line is the current best value of $d=3.8 \pm 0.1$ (Harris et al. 2010).

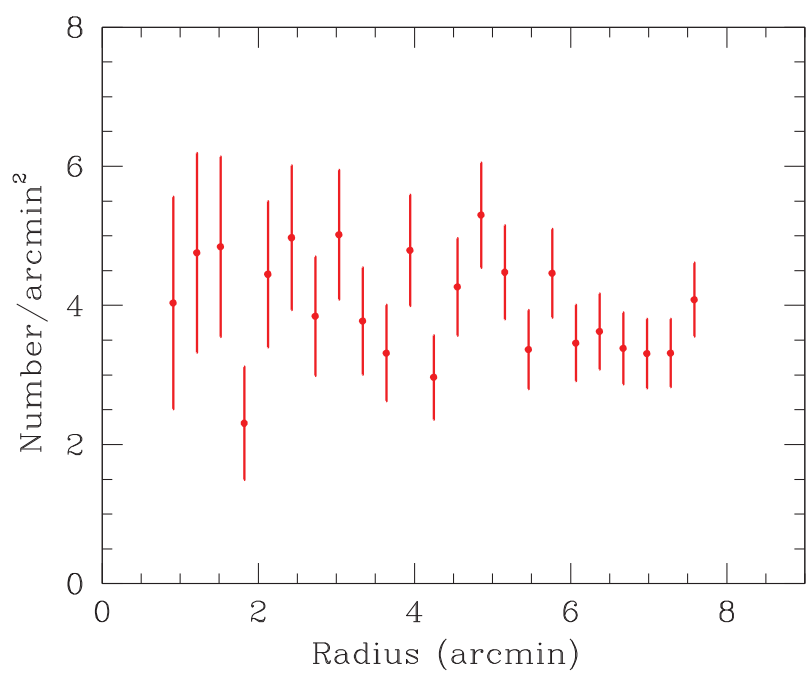

Figure 2 Star counts in NGC 5128 based on data from van den Bergh (1979). 
2004) but they are hard to unambiguously separate from the noise of foreground Milky Way halo stars and compact background galaxies. The two main reasons for this are proximity and low galactic latitude. At a distance of $3.8 \mathrm{Mpc}, 1$ arcmin corresponds to $\sim 1100 \mathrm{pc}$ and, consequently, a survey to discover a widely distributed population like GCs must cover an area of more than two square degrees. Observationally, this is a major challenge, but there are other even more significant challenges.

Since GCs in galaxies well beyond the Local Group generally appear starlike, they cannot be distinguished from either foreground field stars or background galaxies by appearance alone. To reduce contamination, the fact that clusters occupy a well defined range in colour and luminosity is used to detect them photometrically and eliminate a large fraction of non-cluster sources. Unfortunately, for NGC 5128 this is nearly useless. Its brightest GCs should appear at an apparent magnitude of $V \sim 17$ while the peak of the GC luminosity function (GCLF) would be seen at a magnitude of $V \sim 21$. Because NGC 5128 has a low galactic latitude $\left(19^{\circ}\right)$, we see it through the Milky Way halo and disk and its mainly late type stars whose colours and apparent magnitudes will fall in the same range as GC candidates. Compact background galaxies that are fairly easily seen through the dilute halo may also have GC-like colours, and surveys show that their numbers begin to increase rapidly at $V \sim 20-21$, the GCLF peak (Metcalfe et al. 2001). In short, the sample contamination problem is extraordinarily difficult to solve.

The mystery began to be resolved by Graham \& Phillips (1980) with the discovery of a GC candidate $\sim 8.2$ arcmin northeast of the galaxy centre. This was followed quickly by the identification of six more (van den Bergh et al. 1981). All seven were initially selected by their slightly non-stellar appearance and then confirmed spectroscopically as having radial velocities consistent with membership in NGC 5128. Using Schmidt plates with a much larger field of view than the 4-m prime focus plate used by van den Bergh (1979), van den Bergh et al. (1981) also revisited the question of star counts. Their results, reproduced in Figure 3, show a clear excess of objects within $\sim 375$ arcsec of the nucleus; based on this they estimated the total cluster population to be $600 \pm 61$. Later wide field surveys have estimated the total GCS population to be 1000-2000 (Harris et al. 1984; Harris, Harris \& Geisler 2004).

\subsection{GCS Total Population}

Once the existence of GCs in NGC 5128 was established, increased efforts were made to understand this system. The observational issues described above have made it extremely difficult to build an overall picture, as illustrated in the historical overview plotted in Figure 4. Shown plotted vs. year are: estimates of the total GC population (van den Bergh 1979; van den Bergh et al. 1981; Harris et al. 1984; Harris, Harris \& Geisler 2004, and this paper); number of confirmed GCs (Graham \&
Phillips 1980; van den Bergh et al. 1981; Hesser et al. 1986; Rejkuba et al. 2001; Peng, Ford \& Freeman 2004b; Woodley et al. 2005, 2007; Beasley et al. 2008; Woodley \& Gomez 2010b); and, for comparison, the number of confirmed planetary nebulae (Jacoby, Ciardullo \& Ford 1988; Ford et al. 1989; Hui et al. 1993; Peng, Ford \& Freeman 2004a; Walsh et al. 2010). Note that the number of known GCs reaches a plateau at $\sim 100$ in the late 1980s and remains virtually unchanged for more than a decade. This reflects the fact that the 'easy' GC candidates, which could be identified by a slightly non-stellar appearance, had mostly been found. Also remember that adding more to the sample by photometry

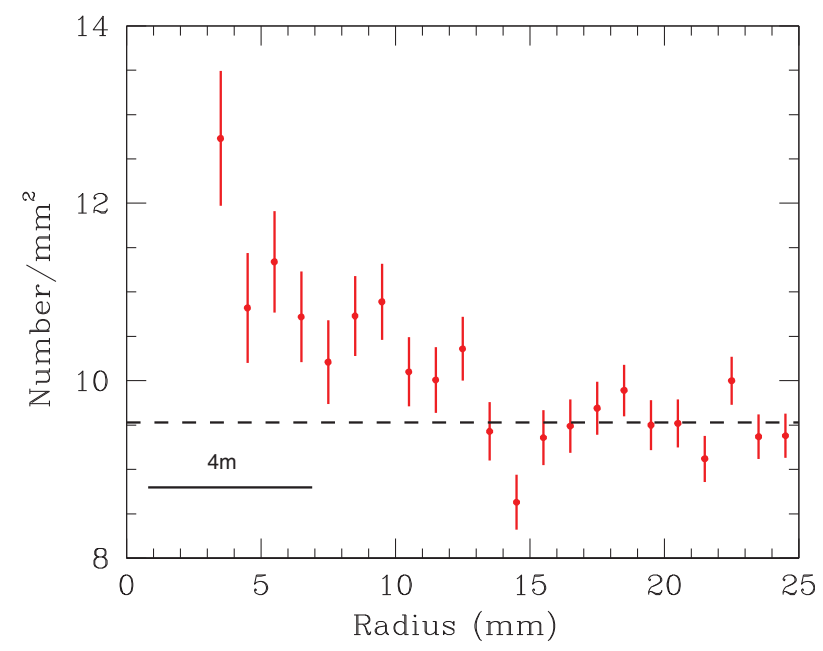

Figure 3 Surface density of objects vs. distance from the centre of NGC 5128, adapted from (Figure 2) van den Bergh (1979); note the line labeled $4 \mathrm{~m}$ indicating the area counted previously by van den Bergh (1979).

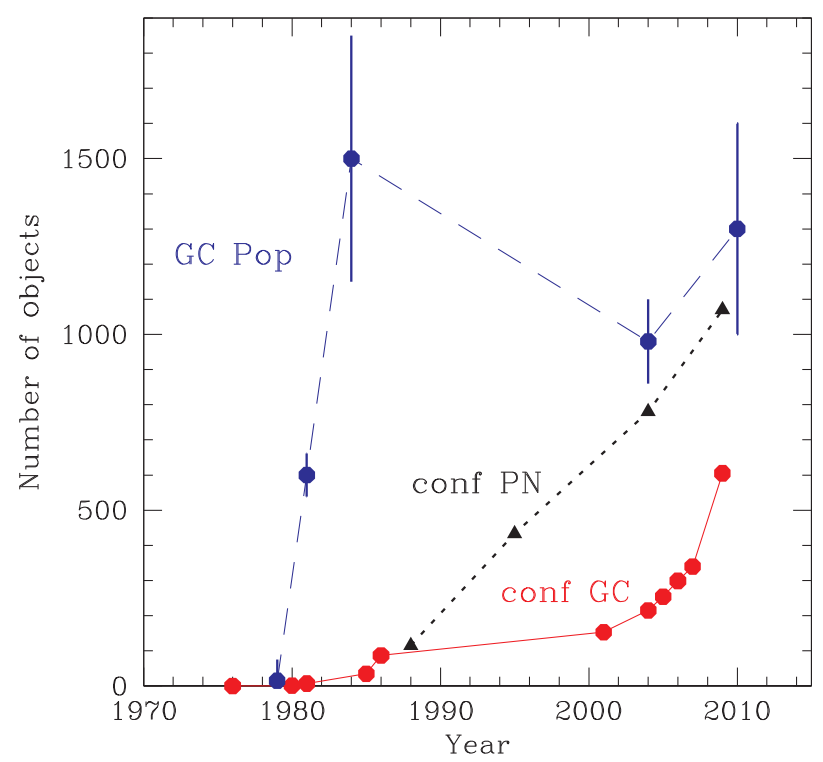

Figure 4 Estimates of total GC population (blue), GCs confirmed by radial velocity and/or resolved by HST imaging, and confirmed planetary nebulae. If the estimated total number of GCs in NGC 5128 is correct (see Figure 5 and accompanying text), then we have now identified approximately half of the GC population. 
alone is an almost impossible task for ground-based seeing, whereas the large angular scale of the system precludes Hubble Space Telescope (HST) imaging due to its small field of view. In their wide field imaging study, Harris et al. (2004) found $\sim 10^{5}$ objects that were in the colour and magnitude range populated by GCs in NGC 5128 . If the total cluster population is $\sim 2000$, it is embedded in a signal to noise ratio $(\mathrm{S} / \mathrm{N})$ of 0.02 ; that is, only about $2 \%$ of the detected star-like objects in the field encompassed by the halo of NGC 5128 are likely to be globular clusters. Recent increases in the number of known clusters have been achieved by the brute force approach of obtaining radial velocity spectra for the brightest candidates (Woodley et al. 2005, 2007, 2010a; Beasley et al. 2008) and the number of confirmed GCs is now 607. Woodley et al. (2010a) fit a Gaussian to the luminosity function (LF) of their sample, finding a turnover of $T_{1}(t o)=19.44 \mathrm{mag}$. This is almost $1 \mathrm{mag}$ brighter than the expected value and a clear sign that this sample is missing most of the fainter clusters. Because both the distance to NGC 5128 and the absolute magnitude of the standard globular cluster luminosity function (GCLF) turnover are well known, we can force a fit to the cluster sample, assuming it is complete for the brightest clusters, and estimate the total population of the cluster system, $N_{t}$. With $(m-M)_{0}=27.9$ (Harris et al. 2010), $A_{V}=0.35$ schlegel (Finkbeiner \& Davis 1998), and $M_{V}($ to $)=-7.3$ for giant ellipticals (Harris 2001), the GCLF turnover should occur at $V=20.95$ or $T_{1}=20.47$. Fitting a Gaussian function with $\sigma=1.4$, typical of $\mathrm{gE}$ galaxies (Harris 2001), yields the result shown in Figure 5, from which we estimate $N_{t}=1300 \pm 300$.

This value is consistent with previous estimates, but is based on a more secure data set, especially for the brightest clusters, and stronger constraints on the distance to NGC 5128. From Figure 5 it is also clear that roughly half

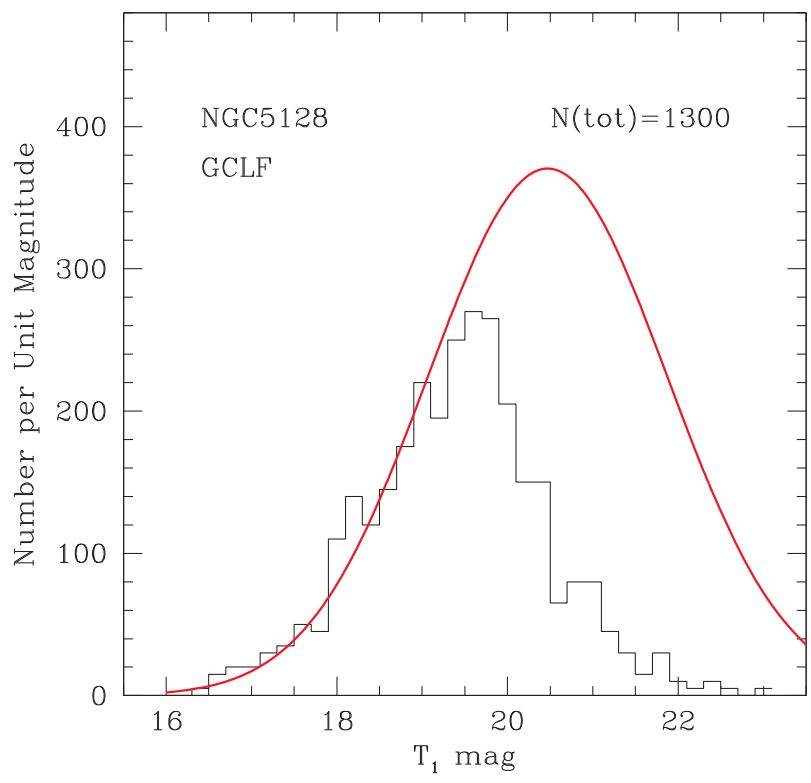

Figure 5 Gaussian function superimposed on the NGC 5128 GC luminosity function using data from Woodley (2010a). the GC population is now known, and that almost all of the undiscovered clusters will be fainter than $V \sim 20$ or $T_{1} \sim 19.5$. Finally, we can calculate the specific frequency $S_{N}=N_{t} 10^{0.4\left(M_{V}^{T}+15\right)}$ (Harris \& Van de Bergh 1981). Combining $N_{t}=1300 \pm 300$ and $M_{V}^{T}=-22.1$ (Harris, Harris \& Geisler 2004), gives $S_{N}=1.9 \pm 0.5$. Disk galaxies typically have low $S_{N} \leq 1.5$, E galaxies have $S_{N}$ ranging from 1 to 10 and even higher, while field E galaxies are on the lower end of this range with $S_{N} \leq 2$ (Harris 2001). Thus $S_{N} \sim 2$ for NGC 5128 is consistent with a normal GCS in a small group.

\subsection{GCS Metallicities and Ages}

Harris et al. (1992) obtained Washington photometry to derive metallicities for $62 \mathrm{GCs}$ based on $C-T_{1}$ colours. The results show a broad, bimodal metallicity distribution with a mean $[\mathrm{Fe} / \mathrm{H}]_{C-T_{1}}=-0.8 \pm 0.2$. None of the clusters shows the extreme (greater than solar) abundances found by Frogel (1984) based on $J H K$ photometry, and it appears likely that these values were due to the difficulty of calibration of colour-metallicity relations at such high abundances.

As the number of confirmed GCs has grown, the 'simple' bimodality of the metallicity distribution function (MDF) has become less clear (e.g., Harris et al. 2004; Peng, Ford \& Freeman 2004b; Beasley et al. 2008; Woodley et al. 2010a, and references therein). But the breadth of the GCS colour distribution and its high mean metallicity have not changed. In addition, we now have chemical abundances and ages based on high $\mathrm{S} / \mathrm{N}$ spectra which are helping to disentangle the frustrating age/ metallicity degeneracy endemic to purely photometric studies. Beasley et al. (2008) and Woodley et al. (2010a) have found that the majority of clusters, both metal-rich and metal-poor, are old ( $>8-10$ Gyr). The GCS has a subpopulation of younger clusters $(<3-5 \mathrm{Gyr})$ but virtually no extremely young members. The clusters are $\alpha$-enhanced

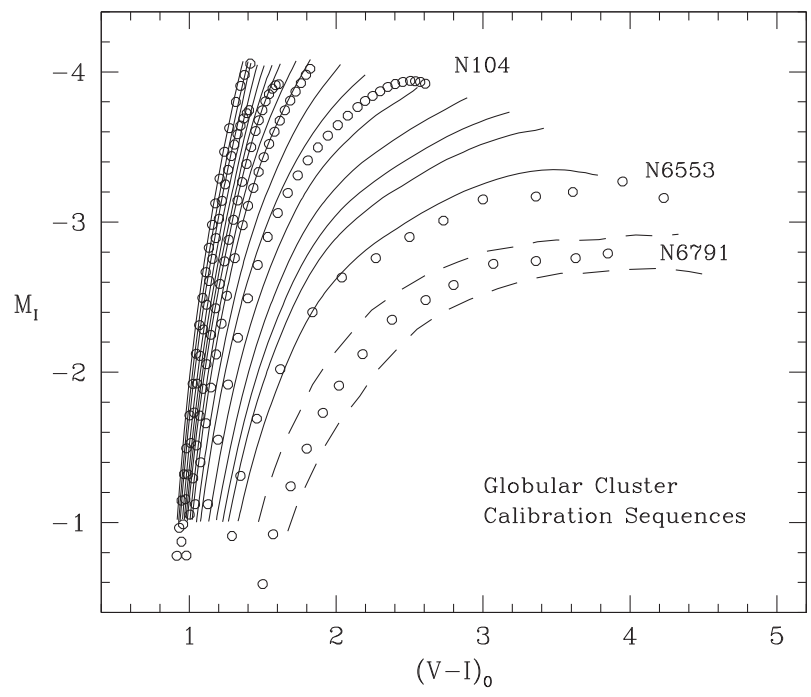

Figure 6 Fiducial giant branches for Milky Way globular clusters ranging in $(\mathrm{m} / \mathrm{H})$ from -2.0 to $\sim$ solar (Harris et al. 2002). 
above solar, but not as strongly as seen for Milky Way globulars.

\subsection{Structure of Globular Clusters}

The structures of Milky Way GCs are well described by single mass, isotropic King models (King 1962) and occupy a narrow range of parameter space similar to the fundamental plane for elliptical galaxies (Djorgovski 1995). McLaughlin (2000) has shown that the clusters have a common core $\mathrm{M} / \mathrm{L}$ and that there is a strong correlation between core binding energy and cluster luminosity. Barmby et al. (2007) and McLaughlin et al. (2008) have shown that the GCs in both M31 and NGC 5128 follow the same fundamental plane for clusters as massive as $\sim 3 \times 10^{6} M_{\odot}$, suggesting that old GCs have similar structural properties in all galaxies. Studies of the most luminous GCs in other systems (Haşegan et al. 2005; Rejkuba et al. 2007) suggest that they bridge the gap between lower mass (normal) clusters and compact dwarfs (UCDs) (Mieske et al. 2008). With a total population about ten times that in the Milky Way, the NGC 5128 GCS is an important resource for exploring how structures and mass-luminosity ratio $(\mathrm{M} / \mathrm{L})$ change for the most massive clusters.

\section{Field Halo Stars}

Knowledge of distant elliptical galaxy haloes relied almost entirely on measurements of their integrated light (see the classic study of NGC 3379 by deVaucouleurs \& Capaccioli 1979). The exceptional angular resolution and sensitivity of the HST, however, provided more knowledge. The first two-colour photometric study of an inner halo field in NGC 5128 (Soria et al. 1996) used images that were not very deep but did show the red giant tip and a broad colour distribution for the giant stars. The large angular size of NGC 5128 helps us in this field of research, and we now have deeper photometry for four fields at distances of $\sim 10,20,30$ and $40 \mathrm{kpc}$ or $\sim 1.5-7 r_{\text {eff }}$ (Harris, Harris \& Poole 1999; Harris \& Harris 2000; Harris et al. 2002; Rejkuba et al. 2005).

\subsection{Metallicity of Halo Stars}

The brightest stars in an old stellar population are the red giants, which first appear at $M_{I} \sim-4.0$. Figure 6, taken from Harris et al. (2002), shows this in the form of fiducial red giant branches (RGBs) for Milky Way GCs covering a metallicity range of $-2 \leq[\mathrm{m} / \mathrm{H}] \leq 0$. From stellar model calculations (e.g., van den Berg et al. 2000) we know the colour of the giant branch varies much less with age than metallicity. Therefore, interpolating with RGB tracks calibrated onto the Milky Way giant branch (GB) grid provides a fast, efficient way to derive a first order MDF. We use $V, I$ photometry because it has a strong metallicity sensitivity for old stars; though not the best colour option, it represents a compromise between the best cameras available and the spectral energy distributions of these old stars.

The $I, V-I$ colour magnitude diagram (CMD) for the outermost ( $\sim 40 \mathrm{kpc})$ NGC 5128 field (Rejkuba et al.
2005 ) is shown in Figure 7. This is the deepest of the halo star data sets but shows much the same GB characteristics as the other three: a well defined upper boundary, especially on the blue (metal-poor) side of the GB and a very wide range in colour. Figure 8 shows the MDFs for all four fields (Rejkuba et al. 2005), with the data for the $\sim 20$ and $30 \mathrm{kpc}$ fields combined because they are essentially indistinguishable (Harris \& Harris 2002). The RGB stars in NGC 5128's halo are predominantly metal-rich with a broad MDF that varies little with galactocentric distance. In all fields, there are almost no metal-poor stars.

The lack of metal-poor stars out to almost $7 r_{\text {eff }}$ was a surprise and leads to the question: where is the classic metal-poor halo in this galaxy? A possible clue can be seen in the $V, I C M D$ of a field centred $\sim 30 \mathrm{kpc}$ or $\sim 12 r_{\text {eff }}$ from the centre of the Leo elliptical NGC 3379. At a distance of $\sim 10 \mathrm{Mpc}$, the HST ACS field covers a wide range in galactocentric distance of $10 \mathrm{kpc}$ or $\sim 3 r_{\text {eff }}$. Unlike what has been found in NGC 5128, the CMD for this field shows a significant metal-poor population along with the metal-rich stars that were expected (Harris et al. 2007). When the image was divided into inner (closer to the galaxy centre) and outer (more distant), the CMDs for these two regions were distinctly different. As seen in Figure 9, the inner field shows a wide RGB colour range which almost disappears in the outer field CMD. Figure 10 shows the radial distributions for the blue $([\mathrm{m} / \mathrm{H}]<-0.7)$ and red $([\mathrm{m} / \mathrm{H}]>-0.7)$ stars superimposed on the surface brightness data from deVaucouleurs \& Capaccioli (1979); the blue population density falls off with radius as $\sigma \sim R^{-1.2}$ and the red as $\sigma \sim R^{-6.0}$.

Is this perhaps the transition to a classic metal-poor halo and, if so, why has this transition not been seen in NGC 5128? The might be because the NGC 5128 data

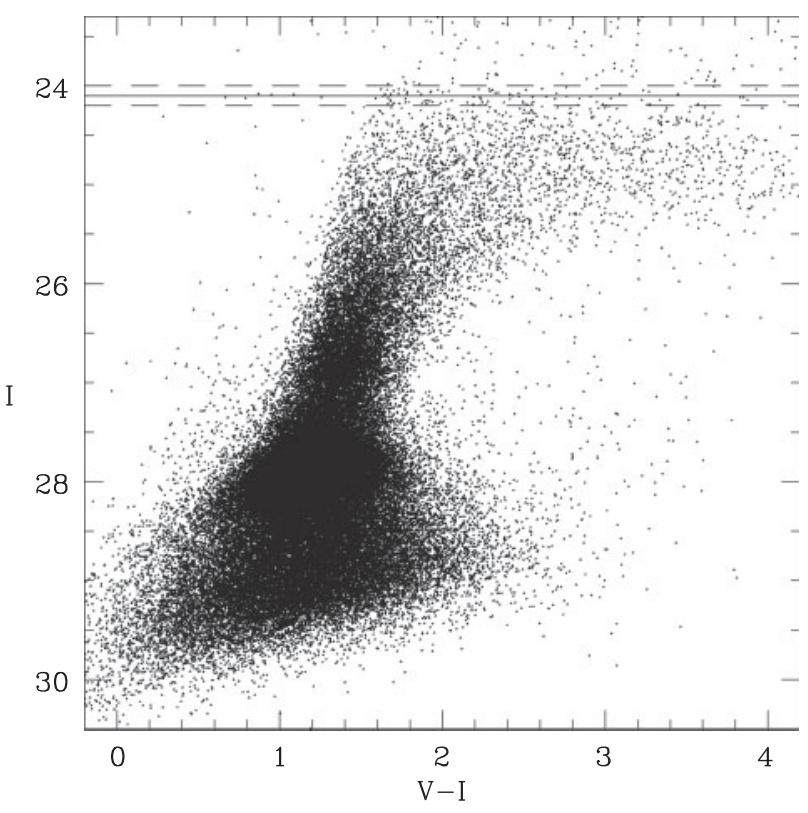

Figure 7 Colour-magnitude diagram for the outer halo of NGC 5128 (Rejkuba et al. 2005), dashed lines showing the TRGB measurement uncertainty of $\pm 0.1 \mathrm{mag}$; compare with GC giant branches plotted in Figure 6. 


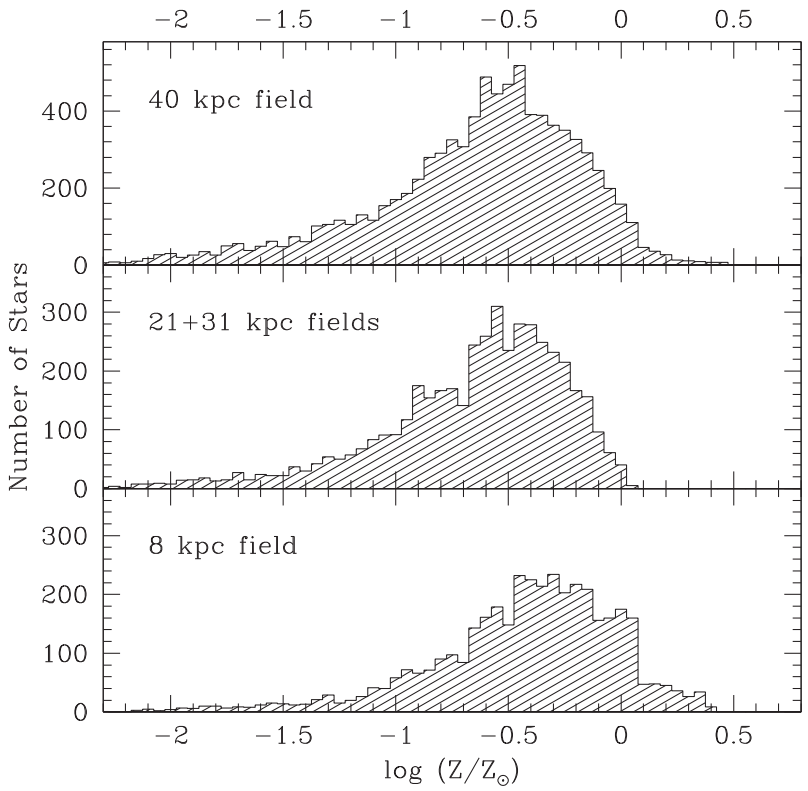

Figure 8 Metallicity distributions for halo fields in NGC 5128 (Rejkuba et al. 2005)

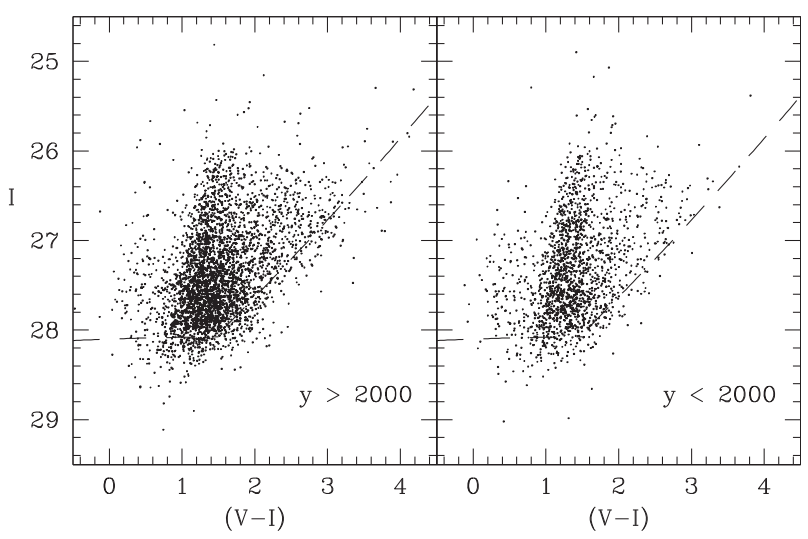

Figure 9 CMD for a halo field in the Leo elliptical NGC 3379 (Harris et al. 2007).

extend to only $\sim 7 r_{\text {eff }}$ compared with the NGC 3379 field which covered a range of $\sim 10.3-13.6 r_{\text {eff }}$. Hence, should we expect the transition from a metal-rich to a metal-poor halo to occur at $\sim 10 r_{\text {eff }}$ ? Observational data which might help answer this question are limited, but a study by Kalirai et al. (2006) appears to have found such a transition for M31. Their $V, I$ photometry for confirmed M31 halo stars shows a radial gradient in metallicity, with the metal-poor component dominating beyond $r>10 r_{\text {eff }}$. $V$, I photometry for a halo field in NGC 5128 beyond $\sim 12 r_{\text {eff }}$ would be a simple way to test this.

\subsection{Age of Halo Stars}

The MDFs for the four halo fields described in the previous section assumed a constant and old ( $\sim 12 \mathrm{Gyr})$ age. Elements of the CMDs, such as the small numbers of stars brighter than the RGB tip and the lack of luminous blue stars, suggest that this is a legitimate starting assumption. However, we would like to have better age constraints for these data, which was the primary motivation for the

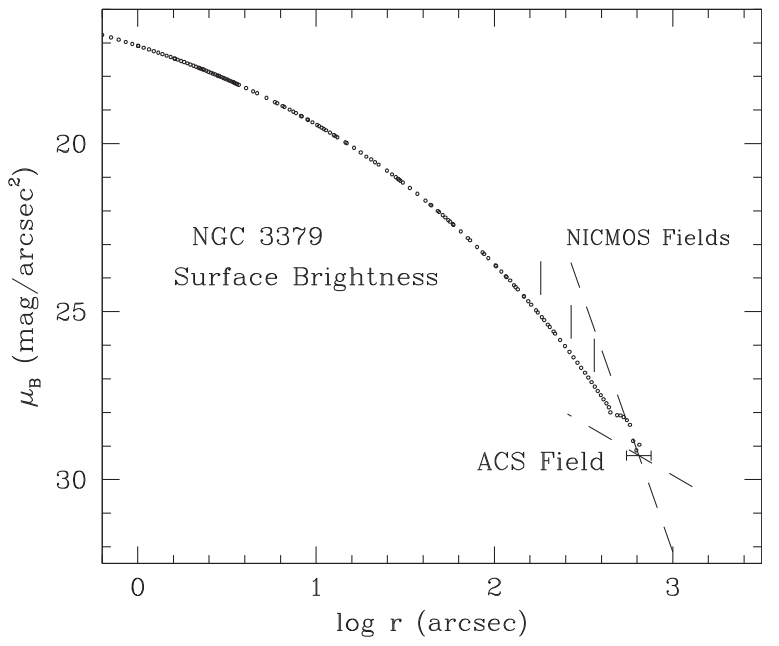

Figure 10 Radial gradients for the blue and red field stars in NGC 3379 from Harris et al. (2007) superimposed on its surface brightness profile from deVaucouleurs \& Capaccioli (1979).

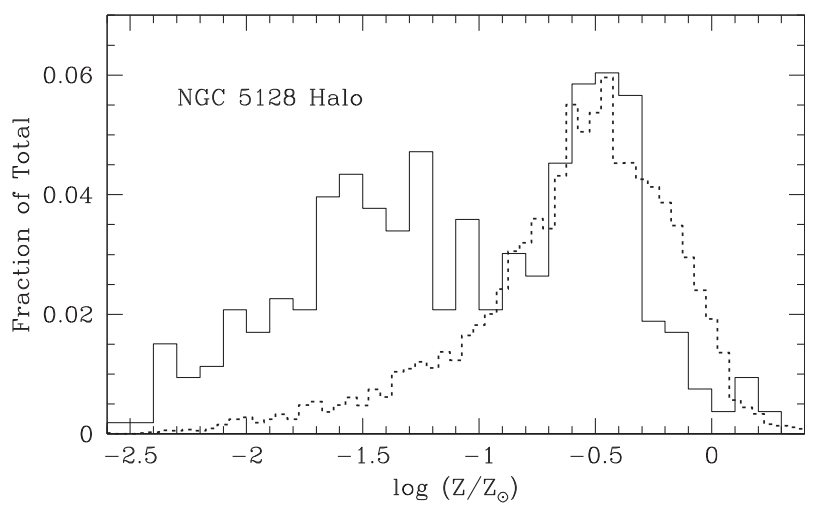

Figure 11 Comparison of GC (solid histogram) and field star (dotted histogram) MDFs in NGC 5128 from Harris (2010).

$40 \mathrm{kpc}$ study (see Figure 7). A CMD that reaches the main sequence turnoff is out of the question at present, but it was possible to obtain data to the depth of the horizontal branch $(I=28-29)$. The resulting photometry is good enough to compare with CMD simulations and narrow the possible range in age and metallicity using red clump and asymptotic giant branch stars as well as those on the RGB. It now appears that the dominant population in this outer field has an age of $\sim 10-12$ Gyr and is probably combined with a small fraction of younger ( $\sim 5 \mathrm{Gyr})$ stars.

A comparison between the $\mathrm{GC}$ and halo field stars (see Figure 11 from Harris 2010) shows some intriguing similarities. The metallicity peaks for the halo stars and the metal-rich GCSs are quite similar and current analysis indicates that both samples are mainly old with a small younger component. Simplistically this suggests that major star formation episodes at $\sim 12$ and 5 Gyr may have occurred in NGC 5128.

\section{Summary}

- There is now agreement, within a few per cent, on the distance to NGC 5128, making it possible to interpret observed properties with new confidence. 
- Although there is still debate as to whether this is an S0p or Ep galaxy, the properties of its halo and individual stars are consistent with classifying NGC 5128 as an Ep galaxy.

- We have a sample of $\sim 600$ GCs confirmed by radial velocity and/or angular resolution, $\sim 50 \%$ of the estimated total population. Roughly half of these are metal-rich and the majority are old.

- In spite of these numbers, global properties of the system are not well known because of major biases in the spatial coverage.

- Observations of the spatial structure of $>200$ GCs shows that they follow the same fundamental plane relation as GCs in the Milky Way and M31. The NGC 5128 system allows us to trace this relation to higher GC luminosity and mass than we can in the Milky Way or M31.

- Over a wide range in galactocentric distance, the halo stars are remarkably similar and predominantly metalrich, and it appears that these metal-rich stars are old, possibly with ages similar to those of the metal-rich GCs. We may need to observe the halo to galactocentric distances twice that of current data sets in order to uncover its metal-poor component.

\section{Acknowledgments}

G.L.H.H. acknowledges financial support through a research grant from the Natural Sciences and Engineering Research Council of Canada. The beginnings of this paper were developed during a visit to ESO Garching, sponsored through the ESO visitor programme.

\section{References}

Baade, W. \& Minkowski, R., 1954, ApJ, 119, 215

Barmby, P., McLaughlin, D. E., Harris, W. E., Harris, G. L. H. \& Forbes, D. A., 2007, AJ, 133, 2764

Beasley, M. A., Bridges, T., Peng, E., Harris, W. E., Harris, G. L. H., Forbes, D. A. \& Mackie, G., 2008, MNRAS, 386, 1443

deVaucouleurs, G. \& Capaccioli, M., 1979, ApJS, 40, 699

Djorgovski, S., 1995, ApJ, 438L, 29

Dufour, R. J., Harvel, C. A., Martins, D. M., Schffer, F. H., III, Talent, D. L., Wells, D. C., van den Bergh, S. \& Talbot, R. J., Jr., 1979, AJ, 84, 284

Ebneter, K. \& Balick, B., 1983, PASP, 95, 675

Ford, H. C., Ciardullo, R., Jacoby, G. H. \& Hui, X., 1989, IAUS, 131,335

Freeman, K. C., 1970, ApJ, 160, 811

Frogel, J. A., 1984, ApJ, 278, 119

Graham, J. A., 1979, ApJ, 232, 60

Graham, J. A. \& Phillips, M. M., 1980, ApJ, 239L, 97

Gomez, M. \& Woodley, K. A., 2007, ApJ, 670L, 105

Harris, W. E., 2001, Saas-Fee Advanced Course 28, Lecture Notes 1998, Swiss Society for Astrophysics and Astronomy, Eds. Labhardt, L. \& Binggale, B. (Berlin: Springer-Verlag), 223

Harris, W. E., 2010, PhilTransRoySoc, 368, 889

Harris, W. E. \& Racine, R., 1997, ARAA, 17, 241

Harris, W. E. \& van den Bergh, S., 1981, AJ, 86, 1627

Harris, G. L. H., Hesser, J. E., Harris, H. C. \& Curry, P. J., 1984, ApJ, 287,175

Harris, G. L. H., Geisler, D., Harris, H. C. \& Hesser, J. E., 1992, AJ, 104,613

Harris, G. L. H., Harris, W. E. \& Poole, G. B., 1999, AJ, 117, 855

Harris, G. L. H. \& Harris, W. E., 2000, AJ, 120, 2423
Harris, W. E. \& Harris, G. L. H., 2002, AJ, 123, 3108

Harris, W. E., Harris, G. L. H., Holland, S. T. \& McLaughlin, D. E., 2002, AJ, 124, 1435

Harris, G. L. H. et al., 2004a, AJ, 128, 712

Harris, G. L. H., Harris, W. E. \& Geisler, D., 2004b, AJ, 128, 723

Harris, W. E., Harris, G. L. H., Layden, A. C. \& Wehner, E. M. H., 2007, ApJ, 666, 903

Harris, G. H., Rejkuba, M. \& Harris, W. E., 2010, PASA, in press (arXiv:0911.3180).

Hașegan, M. et al., 2005, ApJ, 27, 203

Hesser, J. E., Harris, H. C., Harris, G. L. H. \& Lafontaine, A., 1986, BAAS, 18, 902

Hui, X., Ford, H. C., Ciardullo, R. \& Jacoby, G. H., 1993, ApJ, 414, 463

Israel, F. P., 1998, ARAA, 8, 237

Jacoby, G. H., Ciardullo, R. \& Ford, H. C., 1988, ASP 100th Anniversary Symp. Proc., Victoria, Canada, 1998 June 29-July 10 (San Francisco, CA, Astronomical Society of the Pacific), 42

Kalirai, J. S. et al., 2006, ApJ, 648, 389

King, I. R., 1962, AJ, 67, 471

McConnachie, A. W., Irwin, M. J., Ferguson, A. M. N., Ibata, R. A., Lewis, G. F. \& Tanvir, N., 2005, MNRAS, 356, 979

McLaughlin, D. E., 2000, ApJ, 539, 618

McLaughlin, D. E., Barmby, P., Harris, W. E., Forbes, D. A. \& Harris, G. L. H., 2008, MNRAS, 384, 563

Metcalfe, N., Shanks, T., Campos, A., Cracken, H. J. \& Fong, R., 2001, MNRAS, 373, 795

Mieske, S. et al., 2008, AA, 487, 921

Morgan, W. W., 1958, PASP, 70, 364

Peng, E. W., Ford, H. C. \& Freeman, K. C., 2004a, ApJ, 602, 685

Peng, E., Ford, H. C. \& Freeman, K. C., 2004b, ApJ, 602, 705

Rejkuba, M., Minniti, D., Silva, D. R. \& Bedding, T. R., 2001, AA, 379,781

Rejkuba, M., Minniti, D., Silva, D. R. \& Bedding, T. R., 2003, AA, 411, 351

Rejkuba, M., 2004, AA, 413, 903

Rejkuba, M., Greggio, L., Harris, W. E., Harris, G. L. H. \& Peng, E. W., 2005, ApJ, 631, 262

Rejkuba, M., Dubath, P., Minniti, D. \& Maylan, G., 2007, AA, 469,147

Sandage, A., 1961, The Hubble Atlas of Galaxies, Carnegie Institution of Washington Publications 618 (Washington, DC: Carnegie Institution of Washington)

Sandage, A. \& Tammann, G. A., 1981, A Revised Shapley-Ames Catalog of Bright Galaxies (Carnegie Institution of Washington Publ. 635; Washington, DC: Carnegie Institution of Washington)

Schlegel, D. J., Finkbeiner, D. P. \& Davis, M., 1998, ApJ, 500, 525

Sérsic, J. L., 1958, Obs, 78, 24

Soria, R. et al., 1996, ApJ, 465, 79

van den Bergh, S., 1976, ApJ, 206, 883

van den Bergh, S., 1979, PASP, 91, 639

van den Bergh, S., Hesser, J. E. \& Harris, G. L. H., 1981, AJ, 86, 24

van den Bergh, S., 1990, ApJ, 348, 57

van den Bergh, S., 2009

van den Berg, D. A., Swenson, F. J., Rogers, F. J., Iglesias, C. A. \& Alexander, D. R., 2000, ApJ, 532, 430

Walsh, J., Walton, N. A., Jacoby, G. H. \& Priest, E. R., 1999, AA, 346, 793

Walsh, J. R., Rejkuba, M. \& Walton, N. A., 2010, in preparation

Woodley, K. A., Harris, W. E. \& Harris, G. L. H., 2005, AJ, 129, 2654

Woodley, K. A., Harris, W. E., Beasley, M. A., Peng, E. W., Bridges, T. J., Forbes, D. A. \& Harris, G. L. H., 2007, AJ, 134, 494

Woodley, K. A., Harris, W. E., Puzia, T., Gomez, M., Harris, G. L. H. \& Geisler, D., 2010a, AJ, in press (arXiv:1002.3142)

Woodley, K. A. \& Gomez, M., 2010, PASA, in press (arXiv:0911.2552) 